$\bigoplus$ Science and Engineering Research Council

雨

त $\frac{1}{\nwarrow}$ Chilton DIDCOT Oxon OX11 0QX

RAL-93-076

Estimating the Thermal Transport Properties of Building Components

A D Irving S Dudek G Warren and T Dewson

LIBRARY, R61

-6 DEC 1993

RUTHERFORE APQLETON LABORATORY

October 1993 
Science and Engineering Research Council "The Science and Engineering Research Council does not accept any responsibility for loss or damage arising from the use of information contained in any of its reports or in any communication about its tests or investigations" 


\title{
Estimating the thermal transport properties of building components
}

\author{
AD Irving+, S Dudek ${ }^{0}, \mathrm{G}$ Warren ${ }^{0}$ and T Dewson* \\ + Rutherford Appleton Laboratory, Chilton, Oxford, Oxon, OX11 0QX, UK. \\ ${ }^{0}$ Department of Building Science, The University, Newcastle Upon Tyne, NE1 7RU, UK. \\ * Department of Mathematics, University Walk, University of Bristol, Bristol, BS8 1TR, UK.
}

\begin{abstract}
Real building's and their components experience dynamic thermal transmission. It is possible to represent thermal transmission as either a supposition of local constitutive convolution equations in a local region of the solid or as a directed graph network between connected thermodynamic regions within the solid. Both the loacl constitutive convolution and directed graph network representations are based on response factors which can, in principle, be used to estimate dynamic thermal transmission of the building components. The response factors can also be used to predict the performance of the materials under a range of design loadings. These response factors for each representation can be estimated directly from time series data of the physical observables under general stochastic boundary conditions. Indeed, it can be demonstrated that each of the representations can accurately characterise the thermal performance of building components.
\end{abstract}

The purpose of this paper is to show that only the loacl constitutive equations yield, for all cases considered, the correct values for the physical properties of the materials under test, whereas the directed graph network representations are unreliable. This is because directed graph network representations are ill posed for the one dimensional flow case and are likely to lead to erroneous predictions for the design performance of buildings. The loacl constitutive equations allow the measurement of thermal conductivity and transmission of building components in the field. Thus providing a unique insight into the actual thermal performance of constructions in use. 


\section{Introduction}

Buildings do not exist in a steady state world; they respond to the continuously changing weather and the needs of the occupants. To measure the thermal characteristics of these buildings the appropriate analysis techniques need to be employed. This work uses novel time series analysis techniques to determine the thermal characteristics for a closely defined thermal transport problem. It is possible to represent thermal transmission as either a supposition of local constitutive convolution equations in a local region of the solid or as a directed graph network between connected thermodynamic regions within the solid. Both the local constitutive convolution and network representations are based on response factors which can, in principle, be used to estimate dynamic thermal transmission of the building components. Of these, only the local constitutive convolution equation representation based on the response factors was consistently able to determine accurate values for the thermal conductivity of a wide range of building materials.

A specially constructed test facility was used to measure the thermal conductivity of a wide range of common building materials. The experiments were performed for each material under dynamical meteorological boundary conditions. The time series data for both sets of boundary conditions were analysed by two representations and compared to the ratio of mean's method and published data. This enabled the direct comparisons to be made between these representations. 


\section{Representations of the thermal process}

The two representations used in the present work are:

1. the local constitutive representation where the local one dimensional heat flux in the solid material is assumed to be related to the historical local temperature gradient.

2. the directed graph network representation, where the heat flux and temperature in one region of the solid is related to the historical heat flux and temperature in another region.

The moment hierarchy method [1] is used to calculated the response factor values and thermal transport coefficients for each of the representations. A thermostatic network representation relating the thermodynamic fluxes to the thermodynamic forces has recently been developed by Peusner [2-5] and that representation is considered in detail elsewhere [6].

\section{After Onsager}

The Onsager representation considered is concerned with the constitutive equations which relate the local thermodynamic fluxes to the local thermodynamic forces. A thermodynamic flux is characterised in terms of the thermodynamic forces acting and the constants of proportionality are the transport coefficients, for example, in this case the thermal conductivity. In the Onsager representation, a given thermodynamic flux, $\left\{Q_{k}(t)\right\}$, can be written as a linear function of the local thermodynamic forces, $\left\{F_{j}(t)\right\}$, with

$\mathrm{Q}_{\mathrm{k}}(\mathrm{t})=\mathrm{Q}_{\mathrm{k}}\left(\mathrm{F}_{1}, \ldots, \mathrm{F}_{\mathrm{A}}\right)$

The linear steady state transport properties can be described by the irreversible thermodynamic equations of Onsager [7,8]. 
The linear form of the local constitutive equations relate the independent thermodynamic fluxes, $\left\{Q_{k}(t)\right\}$, in terms of their conjugate thermodynamic forces, $\left\{F_{i}(t)\right\}$, and a set of linear steady state Onsager coefficients, $\mathrm{L}_{\mathrm{ik}}$, with

$Q_{k}(t)=\sum_{i} L_{i k} F_{i}(t)$

The most simple case to study is the one dimensional heat conduction experiment. Gurtin and Pipkin [4] developed a realistic field theory for heat conduction using local onstitutive equations with assumptions that lead to finite propagation speeds. The linearised constitutive equation for the heat flux in terms of the local temperature gradient of their field theory

$$
\mathrm{Q}_{\mathrm{k}}(\mathrm{t})=\sum_{\sigma=0}^{\mathrm{t}} \mathrm{L}_{\mathrm{Q}_{\mathrm{k}} \nabla \mathrm{T}}(\mathrm{t}-\sigma) \nabla \mathrm{T}(\sigma)
$$

Chen and Nunziato [10] used the second law of thermodynamics to show that

$$
\kappa=\sum_{\sigma=0}^{\mathrm{t}} \mathrm{L}_{\mathrm{Q}_{\mathrm{k}} \nabla \mathrm{T}}(\sigma)>0
$$

where $\kappa$ is the steady state thermal conductivity of the solid and the response factors, $\mathrm{L}_{\mathrm{Q}_{\mathrm{k}} \nabla \mathrm{T}}(\mathrm{t})$, are the dynamic thermal conductivity values.

\section{After Carsaw and Jaeger}

Carslaw and Jaeger [11] considered the relationship between the heat flux and the temperature values on each surface of a parallel sided homogeneous isotropic slab. They gave linear directed graph network expressions to describe the one dimensional steady relationships between the heat fluxes and temperature fields with the vector convolution as

$\left|\begin{array}{l}\mathrm{Q}_{1}(\mathrm{t}) \\ \mathrm{T}_{1}(\mathrm{t})\end{array}\right|=\left|\begin{array}{ll}\mathrm{K}_{\mathrm{Q}_{1} \mathrm{Q}_{2}}(\sigma) & \mathrm{K}_{\mathrm{Q}_{1} \mathrm{~T}_{2}}(\sigma) \\ \mathrm{K}_{\mathrm{Q}_{1} \mathrm{~T}_{2}}(\sigma) & \mathrm{K}_{\mathrm{T}_{1} \mathrm{~T}_{2}}(\sigma)\end{array}\right|\left|\begin{array}{c}\mathrm{Q}_{2}(\mathrm{t}-\sigma) \\ \mathrm{T}_{2}(\mathrm{t}-\sigma)\end{array}\right|$

where $\sigma$ is the time delay with respect to the present time $t$, and where the time delays are assumed to extend to the finite memory $\mu$ of the conduction process. 
Mitalas and Stephenson [12-14] have also extensively applied the response factor form of the directed graph network approach to the dynamic characterisation of one dimensional conductive thermal flow in the $\mathrm{z}$ domain being given by

$\left|\begin{array}{c}\Theta_{1}(z) \\ \Phi_{1}(z)\end{array}\right|=\left|\begin{array}{cc}A_{\Theta_{1} \Theta_{2}}(z) & B_{\Theta_{1} \Phi_{2}}(z) \\ C_{\Phi_{1} \Theta_{2}}(z) & D_{\Phi_{1} \Phi_{2}}(z)\end{array}\right|\left|\begin{array}{c}\Theta_{2}(z) \\ \Phi_{2}(z)\end{array}\right|$

where $\Theta_{1}(z)$ and $\Theta_{2}(z)$ are the $z$ transformations of the heat fluxes, where $\Phi_{1}(z)$ and $\Phi_{2}(z)$ are the $z$ transformations of the temperatures and where $A_{\Theta_{1} \Theta_{2}}(z), B_{\Theta_{1} \Phi_{2}}(z)$, $\mathrm{C}_{\Phi_{1} \Theta_{2}}(\mathrm{z})$ and $\mathrm{D}_{\Phi_{1} \Phi_{2}}(\mathrm{z})$ are the $\mathrm{z}$ transformations of the time domain response factors in equation (5).

\section{Response factor estimation for the local constitutive representation}

The heat flux, temperature and temperature gradients of sample materials were measured in a calibrated hot box arrangement. From these measurements the dynamic response factors and the thermal conductivity's are estimated using each of the above representations for the thermal process.

The first representation considered is the linear term of a field theoretic description [9] of the one dimensional heat conduction in an isotropic solid which contains no sources of heat. It is assumed that the heat flux observed can be expressed as a convolution between the observed heat flux, $\left\{\mathrm{Q}_{\mathrm{k}}(\mathrm{t})\right\}$, the local temperature gradient, $\{\nabla \mathrm{T}(\mathrm{t})\}$, and the linear response factor, $\mathrm{L}_{\mathrm{Q}_{\mathrm{k}} \nabla \mathrm{T}}\left(\sigma_{1}\right)$. 
For a discrete process, which possesses a finite memory for the thermal conduction process of duration $\mu$, the convolution can be expressed as

$$
\mathrm{Q}_{\mathrm{k}}(\mathrm{t})=\sum_{\sigma_{1}=0}^{\mu} \mathrm{L}_{\mathrm{Q}_{\mathrm{k}} \nabla \mathrm{T}}\left(\sigma_{1}\right) \nabla \mathrm{T}\left(\mathrm{t}-\sigma_{1}\right)
$$

where $\sigma_{1}$ denotes lag. The response factor, $\mathrm{L}_{\mathrm{Q}_{\mathrm{k}} \nabla \mathrm{T}}\left(\sigma_{1}\right)$, is related to the steady state thermal conductivity, $\kappa_{1}$ with

$$
\kappa_{1}=\sum_{\sigma_{1}=0}^{\mu} \mathrm{L}_{\mathrm{J}_{\mathrm{k}} \nabla \mathrm{T}}\left(\sigma_{1}\right)
$$

That is, the area under the response factor between the local heat flux and local temperature gradient is equal to the steady state thermal conductivity. More generally, the area represents the steady state gain between physical observables [1].

A tractable moment hierarchy with well behaved coefficients can be solved for the response factor values [1] with

$$
\mathrm{M}_{\nabla \mathrm{TQ}}\left(\tau_{1}\right)=\sum_{\sigma_{1}=0}^{\mu} \mathrm{L}_{\mathrm{Q}_{\mathrm{k}} \nabla \mathrm{T}}\left(\sigma_{1}\right) \mathrm{M}_{\nabla \mathrm{T} \nabla \mathrm{T}}\left(\tau_{1}, \sigma_{1}\right)
$$

where the cross moment $\mathrm{M}_{\nabla \mathrm{TQ}}\left(\tau_{1}\right)=\sum_{\mathrm{t}=\mu}^{\mathrm{N}} \nabla \mathrm{T}(\mathrm{t}-\tau) \mathrm{Q}_{\mathrm{k}}(\mathrm{t})$ and the auto moment $\mathrm{M}_{\nabla \mathrm{T \nabla T}}\left(\tau_{1}, \sigma_{1}\right)=\sum_{\mathrm{t}=\mu}^{\mathrm{N}} \nabla \mathrm{T}(\mathrm{t}-\tau) \nabla \mathrm{T}(\mathrm{t}-\sigma)$, and where $\mathrm{N}$ denotes the sample length.

Equation (9) is a linear algebra expression $\underline{\mathrm{c}}=\underline{\mathrm{a}} \underline{\mathrm{L}}$, where $\underline{\underline{\mathrm{a}}}$ is the auto moment matrix, $\underline{\mathrm{c}}$ is the cross moment vector and $\underline{L}$ is the vector of response factor values [1]. 


\section{Response factor estimation for the directed graph network representation}

In the Carslaw and Jaeger directed graph network representation, a conducting slab of material both surfaces can simultaneously experience unsteady heat flux and temperature conditions. The two surfaces are assumed to be connected components in the thermostatic network and the heat flux, $\left\{\mathrm{Q}_{1}(\mathrm{t})\right\}$, and the temperature, $\left\{\mathrm{T}_{1}(\mathrm{t})\right\}$, at one surface can be related, by a superposition of convolution equations, to the heat flux, $\left\{\mathrm{Q}_{2}(\mathrm{t})\right\}$, and temperature, $\left\{\mathrm{T}_{2}(\mathrm{t})\right\}$, at the opposite surface by the matrix convolution equation

$\left|\begin{array}{c}\mathrm{Q}_{1}(\mathrm{t}) \\
\mathrm{T}_{1}(\mathrm{t})\end{array}\right|=\left|\begin{array}{ll|}\mathrm{K}_{\mathrm{Q}_{1} \mathrm{Q}_{2}}\left(\sigma_{1}\right) & \mathrm{K}_{\mathrm{Q}_{1} \mathrm{~T}_{2}}\left(\sigma_{1}\right) \\
\mathrm{K}_{\mathrm{T}_{1} \mathrm{Q}_{2}}\left(\sigma_{1}\right) & \mathrm{K}_{\mathrm{T}_{1} \mathrm{~T}_{2}}\left(\sigma_{1}\right)\end{array}\right|$\begin{tabular}{|c}
$\mathrm{Q}_{2}\left(\mathrm{t}-\sigma_{1}\right)$ \\
$\mathrm{T}_{2}\left(\mathrm{t}-\sigma_{1}\right)$
\end{tabular} \mid

The equation for the heat flux is explicitly

$\mathrm{Q}_{1}(\mathrm{t})=\sum_{\sigma_{1}=0}^{\mu} \mathrm{K}_{\mathrm{Q}_{1} \mathrm{Q}_{2}}\left(\sigma_{1}\right) \mathrm{Q}_{2}\left(\mathrm{t}-\sigma_{1}\right)+\sum_{\sigma_{1}=0}^{\mu} \mathrm{K}_{\mathrm{Q}_{1} \mathrm{~T}_{2}}\left(\sigma_{1}\right) \mathrm{T}_{2}\left(\mathrm{t}-\sigma_{1}\right)$

and the steady state thermal conductivity and heat flux gain, $\kappa_{2}$ and $\psi_{2}$, for the Carslaw and Jaeger directed graph network representation are given by

$\mathrm{K}_{2}=\sum_{\sigma_{1}=0}^{\mu} \mathrm{K}_{\mathrm{Q}_{1} \mathrm{~T}_{2}}\left(\sigma_{1}\right)$ and $\psi_{2}=\sum_{\sigma_{1}=0}^{\mu} \mathrm{K}_{\mathrm{Q}_{1} \mathrm{Q}_{2}}\left(\sigma_{1}\right)$

The moment equations to be solved for the response factor values, $\mathrm{K}_{\mathrm{Q}_{1} \mathrm{~T}_{2}}\left(\sigma_{1}\right)$ and $\mathrm{K}_{\mathrm{Q}_{1} \mathrm{Q}_{2}}\left(\sigma_{1}\right)$, in the Carslaw and Jaeger directed graph network representation case are

$\mathrm{M}_{\mathrm{T}_{2} \mathrm{Q}_{1}}\left(\tau_{1}\right)=\sum_{\sigma_{1}=0}^{\mu} \mathrm{K}_{\mathrm{Q}_{1} \mathrm{Q}_{2}}\left(\sigma_{1}\right) \mathrm{M}_{\mathrm{T}_{2} \mathrm{Q}_{2}}\left(\tau_{1}, \sigma_{1}\right)+\sum_{\sigma_{1}=0}^{\mu} \mathrm{K}_{\mathrm{Q}_{1} \mathrm{~T}_{2}}\left(\sigma_{1}\right) \mathrm{M}_{\mathrm{T}_{2} \mathrm{~T}_{2}}\left(\tau_{1}, \sigma_{1}\right)$

and

$\mathrm{M}_{\mathrm{Q}_{2} \mathrm{Q}_{1}}\left(\tau_{1}\right)=\sum_{\sigma_{1}=0}^{\mu} \mathrm{K}_{\mathrm{Q}_{1} \mathrm{Q}_{2}}\left(\sigma_{1}\right) \mathrm{M}_{\mathrm{Q}_{2} \mathrm{Q}_{2}}\left(\tau_{1}, \sigma_{1}\right)+\sum_{\sigma_{1}=0}^{\mu} \mathrm{K}_{\mathrm{Q}_{1} \mathrm{~T}_{2}}\left(\sigma_{1}\right) \mathrm{M}_{\mathrm{Q}_{2} \mathrm{~T}_{2}}\left(\tau_{1}, \sigma_{1}\right)$ 


\section{Ratio of Means Method}

In addition to the two time series representations given above the thermal conductivity of the sample materials will estimated using the ratio of means method and also from the literature. In the ratio of means method steady state conditions are assumed to prevail and the relationship between the local heat flux and the local temperature gradient will be given by the approximation

$$
\sum_{t=1}^{N} Q_{k}(t) \approx-K_{3}\left(\sum_{t=1}^{N} T_{2}(t)-\sum_{t=1}^{N} T_{1}(t)\right)
$$

where $\mathrm{N}$ is the sample length, where it has been assumed that the thickness $\Delta \mathrm{x}=1.0$ and where the conductivity from the ratio of means method, $\kappa_{3}$, is

$$
\kappa_{3} \approx \frac{\Delta x \sum_{t=1}^{N} Q_{k}(t)}{\left(\sum_{t=1}^{N} T_{2}(t)-\sum_{t=1}^{N} T_{1}(t)\right)} \approx U \Delta x
$$

where it is implicit that the heat flux and temperature values are measured in steady state and that the temperature difference used is the actual driving force of the heat flux, and where $\mathrm{U}$ is the thermal transmittance. 


\section{Experimental facility}

The experimental arrangement shown in figure 1 was designed to measure the thermal conductivity of a wide range of medium to low thermal conductivity engineering materials under stochastic boundary conditions. Different sized samples were required to measure the thermal conductivity, the dimensions for each material type were determined using a two dimensional finite element model was used. This allowed rapid optimisation of sample size and establishing the best positions of the sensors.

Essentially the rig consists of a copper heat pipe to atmospheric conditions and another copper heat pipe to a controlled cold temperature bath. The cold bath is an enclosed copper heat exchanger that has cold water pumped through it. The cooling of the water is achieved by using a commercial water chiller. The water is re-circulated through the chiller. The water temperature is fixed at $10.0+/-0.02^{\circ} \mathrm{C}$. The absolute temperatures are measured by carefully calibrated Platinum resistance thermometers and the heat fluxes are measured with heatflow mats. The temperature and heat flux are measured at positions above and below the as shown in figure 1. The test section is surrounded by loose vermiculite insulation. The insulation is contained within a $500 \mathrm{~mm}$ square box. The absolute temperatures at the surface of the samples are measured with carefully calibrated thermo-couples and the heat fluxes are measured with heatflow mats.

The readings are taken every ten seconds over an eleven hour period, the time interval for data collection being determined by the response time of the sensors used. During the test period 4,000 sets of measurements are taken. Of these 4000 points, some 2000 are used to estimate the response factor values of the process and the remaining 2000 points are used to compare with the values of the heat flux predicted using the estimated response factor values. 


\section{Analysis of the data}

The thermal conductivity of each sample was determined using the two formalisms and the ratio of means method. These estimated values are then used to predict the future behaviour of the heat flux at the surface of the sample. These predicted heat flux values are then compared with the actual observed values.

The conductivity values estimated using the local constitutive and the directed graph network representations are compared with the values obtained using the ratio of means method and also from the literature [15].

These estimated response function values are used to predict the future behaviour of the material. The response factor values obtained using the time series techniques can then be used to provide a prediction of the local heat flux, $\left\{\mathrm{Q}_{\mathrm{p}}(\mathrm{t})\right\}$, field which may be compared with the measured heat flux, $\left\{Q_{1}(t)\right\}$, which were not used to estimate the transport coefficients. This provides a sensitive measure of the quality of the response factor characterisation of the thermal transport process, both for the region of data which were used to estimate the response factor values and for those regions of data that were not used to estimate the response factor values. The accuracy of the modelling ability was determined by dividing the mean difference between actual, $\left\{Q_{1}(t)\right\}$, and predicted, $\left\{Q_{p}(t)\right\}$, time series sequences by the variance between them, that is, the students $t$-test is used for the test statistic.

In all cases the values of the test statistics for the differences between the measured, $\left\{Q_{1}(t)\right\}$, and predicted, $\left\{\mathrm{Q}_{\mathrm{p}}(\mathrm{t})\right\}$, output heat flux for both modelled and predicted data lay well within the acceptance region. This means that each of the representations can accurately describe the observed behaviour of the heat flux. 
The important thing is to determine if the coefficient values have any physical meaning. In this case, if the coefficient values are related to the thermal conductivity and U-value of the material. In addition, the robust and consistent nature of the coeficients needs to be established.

The values of the estimated thermal transport conductivities are presented in table 1 . The experimental uncertainties given are dominated by the calibration accuracy of the heat flux mats.

Table 1: Estimated thermal conductivities of a range of materials

\begin{tabular}{|l|l|l|l|l|l|}
\hline & $\begin{array}{l}\kappa_{1} \\
\mathrm{~W} / \mathrm{m}{ }^{\circ} \mathrm{K}\end{array}$ & $\begin{array}{l}\mathrm{K}_{2} \\
\mathrm{~W} / \mathrm{m}^{\circ} \mathrm{K}\end{array}$ & $\begin{array}{l}\psi_{2} \\
\text { flux gain }\end{array}$ & $\begin{array}{l}\mathrm{K}_{3} \\
\mathrm{~W} / \mathrm{m}^{\circ} \mathrm{K}\end{array}$ & $\begin{array}{l}\text { Literature } \\
\text { value }\end{array}$ \\
\hline stainless & 12.20 & 1.88 & 0.039 & 12.19 & 15.00 \\
\pm 0.5 & \pm 0.05 & \pm 0.020 & \pm 0.5 & \pm 0.6 \\
\hline marble & 1.68 & 2.78 & -0.68 & 1.68 & 2.00 \\
& \pm 0.18 & \pm 0.40 & \pm 0.22 & \pm 0.18 & \pm 0.20 \\
\hline glass & 0.827 & 0.005 & 1.007 & 0.826 & 1.0 \\
& \pm 0.04 & \pm 0.002 & \pm 0.05 & \pm 0.04 & \pm 0.05 \\
\hline plaster & 0.200 & 0.295 & 0.586 & 0.199 & 0.17 \\
board & \pm 0.01 & \pm 0.03 & \pm 0.04 & \pm 0.01 & \pm 0.01 \\
\hline Insulating & 0.0277 & 0.164 & 0.085 & 0.0277 & 0.028 \\
foam & \pm 0.002 & \pm 0.01 & \pm 0.009 & \pm 0.003 & \pm 0.002 \\
\hline
\end{tabular}


As can be seen the local constitutive representation gives correct, accurate and consistent values for the conductivities over the whole range of materials considered. In contrast the Carslaw and Jaeger directed graph network representation fails to provide accurate or consistent values for the building materials considered here. The reason that the directed graph network representation is not consistent and fail to give the correct answers for the thermal transport coefficients can be seen from the basic formulation of the problem, in that it is assumed the functional relationship is of the form

$Q_{2}(t)=f\left(Q_{1}, T_{1}, t\right)$

As the heat flow is approximately one dimensional through the slab of material then $\mathrm{Q}_{2}(\mathrm{t}) \approx \mathrm{Q}_{1}(\mathrm{t})$ and the equation is ill posed, as it can be written as

$\mathrm{Q}_{2}(\mathrm{t}) \approx \mathrm{Q}_{1}(\mathrm{t})+\delta\left(\mathrm{Q}_{1}, \mathrm{~T}_{1}, \mathrm{t}\right)$

In table two the estimated conductivity for four different plasterboard samples are given.

Table 2: Estimated thermal conductivity of four plasterboard samples

\begin{tabular}{|l|l|l|l|l|l|}
\hline Plasterboard & $\begin{array}{l}\kappa_{1} \\
\mathrm{~W} / \mathrm{m}^{\circ} \mathrm{K}\end{array}$ & $\begin{array}{l}\kappa_{2} \\
\mathrm{~W} / \mathrm{m}^{\circ} \mathrm{K}\end{array}$ & $\begin{array}{l}\Psi_{2} \\
\text { flux gain }\end{array}$ & $\begin{array}{l}\kappa_{3} \\
\mathrm{~W} / \mathrm{m}^{\circ} \mathrm{K}\end{array}$ & $\begin{array}{l}\text { Literature } \\
\text { value }\end{array}$ \\
\hline 1 & 0.215 & 0.126 & 0.605 & 0.214 & 0.17 \\
\pm 0.01 & \pm 0.710 & \pm 1.37 & \pm 0.01 & \pm 0.01 \\
\hline 2 & 0.217 & -0.52 & 1.97 & 0.217 & 0.17 \\
& \pm 0.01 & \pm 0.16 & \pm 0.30 & \pm 0.01 & \pm 0.01 \\
\hline 3 & 0.219 & 0.005 & 1.06 & 0.215 & 0.17 \\
& \pm 0.01 & \pm 0.003 & \pm 0.04 & \pm 0.01 & \pm 0.01 \\
\hline 4 & 0.200 & 0.295 & 0.586 & 0.199 & 0.17 \\
\hline
\end{tabular}


As can be seen the local constitutive representation gives correct, accurate and consistent values for the conductivities over the whole range of materials considered. In contrast the Carslaw and Jaeger directed graph network representation fails to provide consistent values for the building materials considered here.

Current many of the thermal transport calculation procedures for buildings are based on the Carslaw and Jaeger directed graph network representation. This being the case, is it very likely that building designs based on them will not accurately represent of the true performance of the actual building, see for example [16] which shows large discrepancies between the measured and the modelled thermal performance of a wide range of building construction types. 


\section{Conclusions}

In this work the thermal transport coefficients for a range of different building materials have been determined using the local constitutive and the Carslaw and Jaeger directed graph network representations. Each of the representations were able to accurately characterise the measured heat flux. However, only the local constitutive representation gave consistent and accurate values for the materials examined. Although the Carlsaw and Jaeger directed graph network and the representation could produce reasonable thermal transport coefficient values for some cases, it was shown not to be accurate or consistent in general. For this reason, designs based on the Carlsaw and Jaeger directed graph network representation and not likely to accurately represent the actual performance of the building. As many of the current calculation procedures are based on the Carslaw and Jaeger directed graph network representation it is recommended the further studies be undertaken to develop alternative procedures and extend the experimental studies to cover a wider range of materials, composites and building structures operating in real meteorological conditions.

\section{Acknowledgements}

The authors would like to acknowledge that this work was funded by the UK Science and Engineering Research Council. 


\section{References}

[1] Irving A D, Stochastic Sensitivity Analysis, Applied Mathematical

Modelling, Vol. 16, January, 1992, p 3-15.

[2] Peusner L, Network thermostatics, J. Chem. Phys., Vol. 83, No. 3, 1985, p 1276.

[3] Peusner L, A network thermostatic approach to Hill and King-Altman reaction diffusion kinetics, J. Chem. Phys., Vol. 83, No. 11, 1985, p 5559-5566.

[4] Peusner L, Global reaction: Diffusion coupling and reprocity in linear asymmetric kinetic networks, J. Chem. Phys., Vol. 77, No. 11, 1982, p 5500-5507.

[5] Peusner L, Studies in network thermodynamics, Elsevier, Amsterdam, The Netherlands, 1986.

[6] Irving $\mathrm{AD}$, Dudeck $\mathrm{S}$, Dewson $\mathrm{T}$ and Warren $\mathrm{G}$, On the multidimensional convolution and graph network representations of thermodynamic processes, Submitted to J. Phys. Chem., Nov. 1993.

[7] Onsager L, Reciprocal relations in irrervisible processes, I, Phys. Rev., Vol. 37, 1931 , p 405.

[8] Onsager L, Reciprocal relations in irrervisible processes, II, Phys. Rev., Vol. 38, 1931, p 2665.

[9] Gurtin M E and Pipkin A C, A general theory of heat conduction with finite speed waves, Arch. Ration. Mech. Anal, 31, 1968, p 113.

[10] Chen $P$ and Nunziato J, Thermodynamic restrictions on the relaxation functions of the theory of heat conduction with finite speed waves, Z. Angew. Math. Phys., 25, 1974, p 791. 
[11] Carslaw H S and Jaeger J C, Conduction of heat in solids, Clarendon Press, Oxford, 1959.

[12] Mitalas G P and Stephenson D G, Calculation of Heat Flows Through Walls and Roofs, ASHRAE TRANS., Vol. 74, 1968, p 182.

[13] Mitalas G P and Stephenson D G, Room Thermal Response Factors, ASHRAE TRANS., Vol. 73, 1967.

[14] Stephenson D G and Mitalas G P, Cooling Load Calculations by the Thermal Response Factor Method, ASHRAE TRANS., Vol. 73, 1967.

[15] Clarke J A, Yanghe P P and Pinney A A, The harmonisation of thermal properties of building materials, BEPAC research report, 1991.

[16] ASHRAE research project 515-RP, Dynamic heat transmission characteristics of seven generic wall types, Private Communication. 


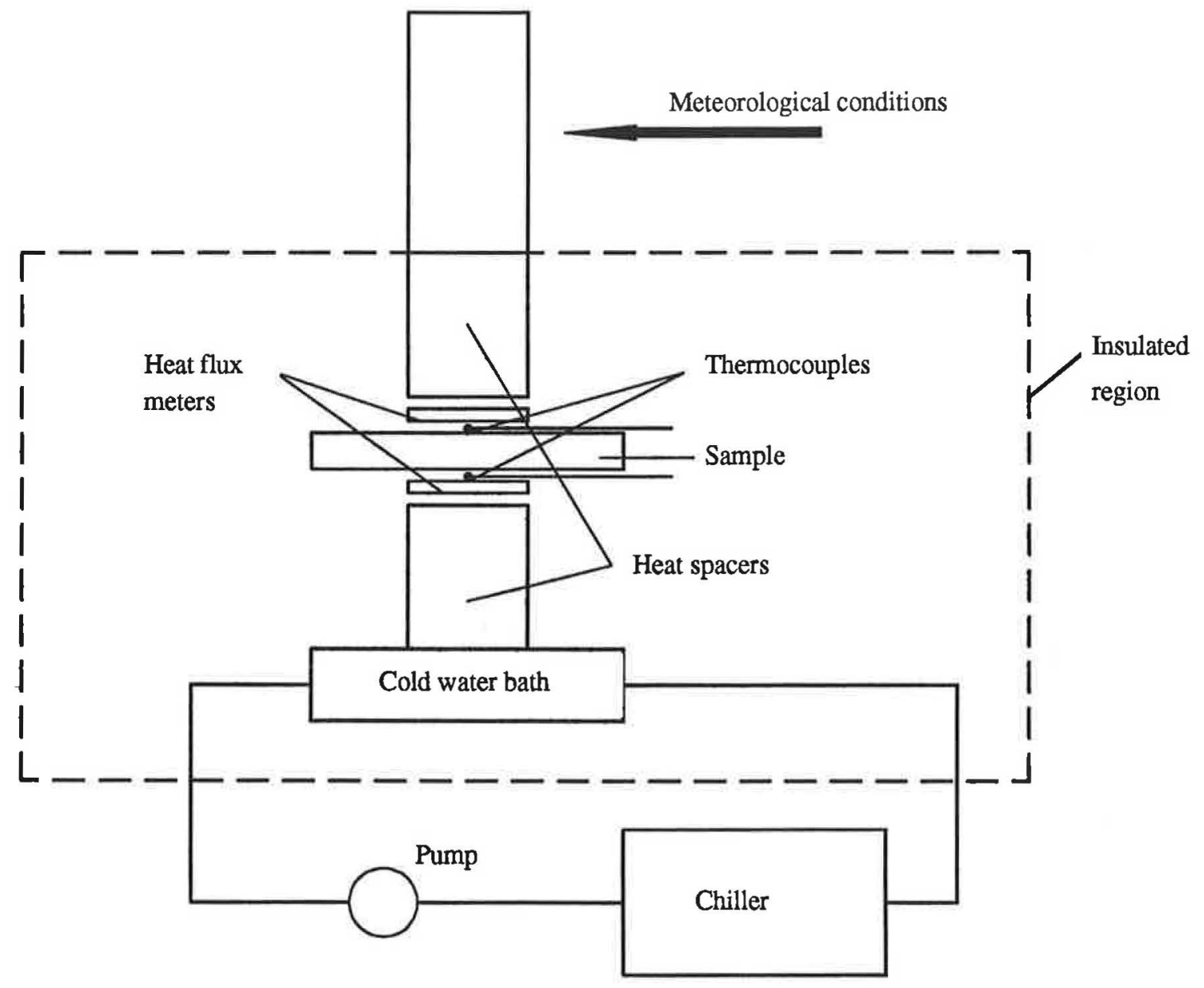

Figure 1. A schematic diagram of the experimental arrangement. 

